\title{
The Protein-Protein Interaction Networks of Dendritic Spines in the Early Phase of Long-Term Potentiation
}

Anna L Proskura, Aleksander S Ratushnyak and Tatyana A Zapara*

Design Technological Institute of Digital Techniques SB RAS, Novosibirsk, Russia

\begin{abstract}
The neuron is a basic element of brain networks. Changes of nerve cell excitability, the conduction of excitation, synaptic memory-forming in the case of the temporal coincidence of synaptic events are the obvious functions of the neuron - element of brain networks. The implementation of the neuron function depends on actions of its numerous molecular systems. The generalization of the complex processes of emergence of synaptic memory, that occur even in separate neuronal compartments, without special tools is a difficult, if at all possible, task. A technology that combines the creation of databases (elements and their relationships) with a visual representation in the form of networks facilitates this process. The developed protein-protein interaction network in dendritic spines of hippocampal pyramidal neurons facilitates the synthesis of numerous experimental data in conceptual knowledge about the principles and molecular mechanisms of neurons functioning.
\end{abstract}

Keywords: Early-phase long-term potentiation; CA1 pyramidal neurons of the hippocampus; Dendritic spines; Glutamate receptors; Small gtpases; Actin-binding proteins; Protein-protein interaction networks

\section{Introduction}

In course of evolution the development of a brain correlates with complication of a molecular nerve cell organization [1-3]. The activity of a neuron entails changes in its connections with other neurons as well as in its molecular networks. Over the last decade notion about the synapse as a relatively simple structure has transformed. Synapses are highly dynamic structures. Their molecular composition is altered in an activity-dependent manner, which modulates the efficacy of subsequent synaptic transmission events. The synapse is a zone of the contacts between cells, transmitting the information and processing it by detecting specific patterns of neural activity and converting this electrical activity into intracellular biochemical events that change the properties of the neuron [4]. Dendritic spines are the postsynaptic component of glutamatergic synapses that originates from an axial dendrite [5]. Dendritic spines are the structure of the excitatory synapse majority of a mammal brain. The flux of ions into postsynaptic terminal of excitatory synapse is regulated by tuning the distribution of receptors and their subunit composition (synaptic plasticity) [57] as well as tuning the morphology of dendritic spines (structural plasticity) [8]. Learning and memory are associated with changes of synaptic modulations that take place in the postsynaptic dendritic part of synapse [9]. The hippocampus is a brain structure involved in short-term memory and the phenomenon of long-term potentiation (LTP) (increases the efficiency of synaptic transmission after an intense and short-term release of neurotransmitter) is the dominant cellular model of learning and memory [10]. Proteomic studies have identified hundreds of postsynaptic proteins organized through physical interactions into multiprotein complexes and networks [11].

The implementation of the neuron function depends on actions of its numerous molecular systems. The generalization of the complex processes of emergence synaptic memory, that occur even in separate neuronal compartments, without special tools designed for formalized description and automated visualization of molecular networks is a difficult, if at all possible, task.
The main purpose of this review is a reconstruction of molecular network of the dendritic spine of pyramidal neurons of the CA1 hippocampal region of rodent, and the action sequence of functional (executive) systems of the neuron involved in synaptic modulations in the early phase of long-term potentiation.

\section{The Functional Systems of the Neuron Involved in Synaptic Modulations in the Early Phase of LTP}

Dendritic spines are the postsynaptic component of glutamatergic synapses that originates from an axial dendrite (button less than $\left.1 \mu \mathrm{m}^{3}\right)$. Dendritic spines are the structure of the majority excitatory synapse of a mammal brain. Molecular networks of the dendritic spine of pyramidal neurons of the CA1 hippocampal region of rodent in the early phase of LTP have been reconstructed using GeneNet computer system [12-15]. The protein-protein interaction (PPI) network of dendritic spines is presented on the site (http://wwwmgs.bionet. nsc.ru/mgs/gnw/genenet/viewer/Early long-term potentiation.html (PPI1)) Datasets of elementary units of the protein-protein interaction networks were made according to the expression of genes encoding proteins (mRNA) of pyramidal neurons of the CA1 hippocampal region of the adult rodent (mouse predominantly). We have used the experimental information which has been manually extracted from the published articles (PubMed) or databases (Swiss-Prot, EMBL, MGI, GeneCard, and TRRD). Journal publications which contain data about the behavior of molecules of dendritic spines of pyramidal neurons of the CA1 hippocampal region in the early phase of longterm potentiation have been mainly used.

*Corresponding author: Tatyana A Zapara, Design Technological Institute of Digital Techniques SB RAS, Novosibirsk, Russia, Tel: +7 383 3308918; E-mail: zapara.tatiana@gmail.com

Received December 20, 2013; Accepted January 08, 2013; Published January 10, 2014

Citation: Proskura AL, Ratushnyak AS, Zapara TA (2014) The Protein-Protein Interaction Networks of Dendritic Spines in the Early Phase of Long-Term Potentiation. J Comput Sci Syst Biol 7: 040-044. doi:10.4172/jcsb.1000136

Copyright: (c) 2014 Proskura AL, et al. This is an open-access article distributed under the terms of the Creative Commons Attribution License, which permits unrestricted use, distribution, and reproduction in any medium, provided the original author and source are credited. 
Citation: Proskura AL, Ratushnyak AS, Zapara TA (2014) The Protein-Protein Interaction Networks of Dendritic Spines in the Early Phase of LongTerm Potentiation. J Comput Sci Syst Biol 7: 040-044. doi:10.4172/jcsb.1000136

In this schemes reflected the interactions between network objects (green arrows) and various the influence reactions (red arrows on influence; dark arrows - off influence; pink arrows - increase influence; blue arrows - decrease influence) (Figure 1). PPI1 network contain the descriptions for 58 proteins (Mus musculus) in GeneNet database format. In this reflects the organization of dendritic spine in one of the possible states. In constructing PPI1 network, we have taken into account the ordered structure of spine with a specific horizontal and vertical compartmental organization at all levels: the membrane (synaptic, perisynaptic, extrasynaptic membrane (EM)); the submembrane (submembrane proteins and postsynaptic density (PSD)); the cytoplasm (the network objects interactions, the receptor intracellular store vesicles (recycling endosome), the receptor transporting vesicles (endocytic vesicle) (Figure 1). Each compartment contains the specific protein set, their functionality defined of synaptic activity.

Proteins that anchor the receptors and bind them to the cytoskeleton (scaffold proteins), protein kinases, proteases, and GTPases form the postsynaptic density (PSD) located under the synaptic membrane [16]. Scaffold proteins control the size and stability of the PSD. These proteins have one or several short conserved sequences of amino acids, called PDZ microdomains. PDZ-microdomains perform high affinity interactions between proteins (not enzymatic). Scaffold proteins form an orthogonal framework of vertical (mostly protein PSD95, SAP97, and S-SCAM) and horizontal (primarily proteins Shank and GKAP) filaments [17]. PSD anchors glutamate receptors in the active area of a dendritic spine.

Glutamate receptors are receptor-channel complexes. The classification of these receptors is based on their sensitivity to $\mathrm{N}$-methyl-D-aspartate and (NMDA) and $\alpha$-amino-3-hydroxyl-5methyl-4-isoxazole-propionate (AMPA). In mature hippocampal neurons, AMPA receptors (AMPAR) comprise two of three subunits (GluR1-3), such as AMPAR type 1/2 (GluR1/GluR2) and AMPAR type 2/3 (GluR2/GluR3) [18]. AMPARs are permeable to $\mathrm{Na}^{+}$. NMDARs are permeable to $\mathrm{Ca}^{2+}$, have chemo- and potential-sensitivity. $\mathrm{Mg}^{2+}$ blocks the ability of NMDAR to bind glutamate at the resting potential (this block is removed by depolarization) [19].

NMDARs are directly anchored at the center of the PSD and AMPAR are anchored through a family of stargazin proteins at the periphery $[20,21]$. In PSDs there are the physical interactions between molecules and positioning the partner molecules in complexes (micro microdomains). AMPARs and NMDARs form a connection with a specific set of proteins in PSD [10].

The dendritic spine cytoskeleton comprises a polymeric (F-actin) and monomeric (G-actin) actin, and F-actin is dominant. A family of small GTPase proteins and actin-binding proteins regulate spinal morphology and synaptic function by modulating reorganization and F-actin turnover. Actin-binding proteins regulate the disassembling of actin microfilaments, the formation of growth complexes, branching and stabilizing of filaments. Dimers of myosin II support the actomyosin

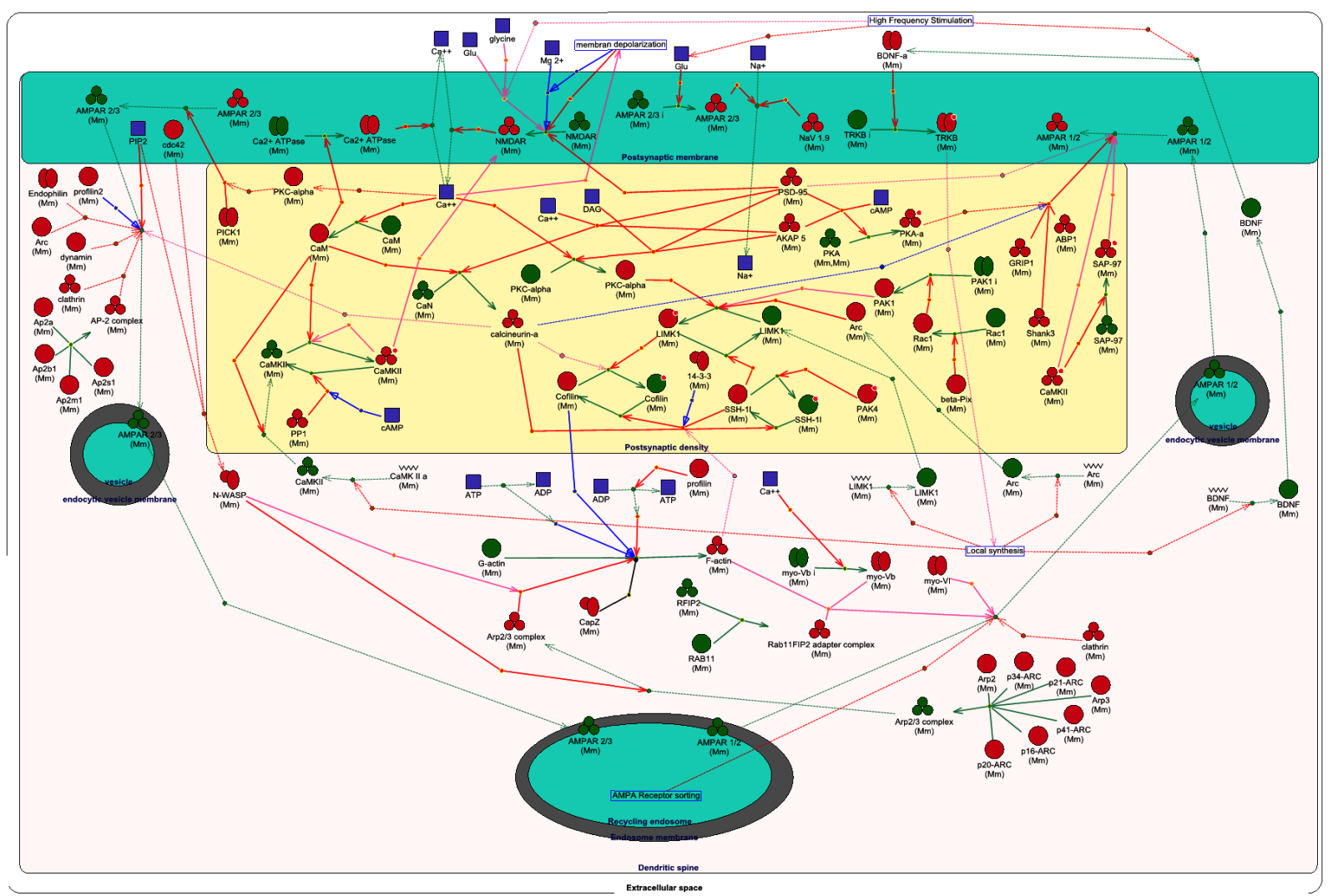

Figure 1: Fragment of network (Early long-term potentiation) (screenshot)). Fragment of network reflecting the basic mechanisms which provide the translating of the neurotransmission to the dendritic spine cytoarchitecture change. Main processes (blue rectangles): inductions (High Frequency Stimulation, membrane depolarization) and expression (breaking of the submembrane actin network - form actomyosin complexes, cross-linking and contractile of actin filaments; form of new the submembrane actin network - elongation and branching of actin filaments). 
tension of the actin cytoskeleton. Transport myosins relocate endo and exovesicles in the spine. Cytoskeletal reorganization in the spines is triggered by mediators through receptor activation (Figure 2).

Transduction of signals from receptors to the actin cytoskeleton is mediated by the small GTPase family, protein regulators of small GTPases, kinases, phosphatases, and numerous regulatory proteins that directly interact with G- and F-actin. The Rho family of small GTPase, such as RhoA, Rac1, and Cdc42, are well represented in PSD and are included in the functional microdomains of glutamate receptors, e.g., small GTPase RhoA is anchored to NMDAR (through scaffold proteins S-SCAM) [22]. This relationship depends on the level of receptor activation: the more the receptor is activated, the weaker is the relationship [23]. Small GTPases circulate between two states: active - GTP-bound (guanine triphosphate) and inactive - GDPbound (guanine diphosphate). Transitions are regulated by guanine nucleotide-exchange factors (GEFs), which promote the transition of GDP into GTP, and GTPase activating proteins (GAPs) promote hydrolysis of guanine nucleotides and the transition of GTP into GDP. Each Rho small GTPase has a specific $\mathrm{GEF}_{\mathrm{Rho}}$ and GAP $\mathrm{Rh}_{\text {. GEFs }}$ and GAPs are present in PSDs where they are ideally situated to be regulated by glutamate receptors and calcium/calmodulin dependent protein kinaseII (CAMKII) or recruit glutamate receptors into spines and PSDs in response to high levels of $\mathrm{Ca}^{2+}$ [24]. For example, our network is shown that the small GTPase Racl is activated by the GEF Tiam $1_{\text {Racl }}$ and inactivated by the GAP MEGAP $P_{\mathrm{Rac}}$, and is indirectly regulated through SynGAP ${ }_{\text {Ras }}$. The NMDAR- $\mathrm{Ca}^{2+}$-CAMKII cascade

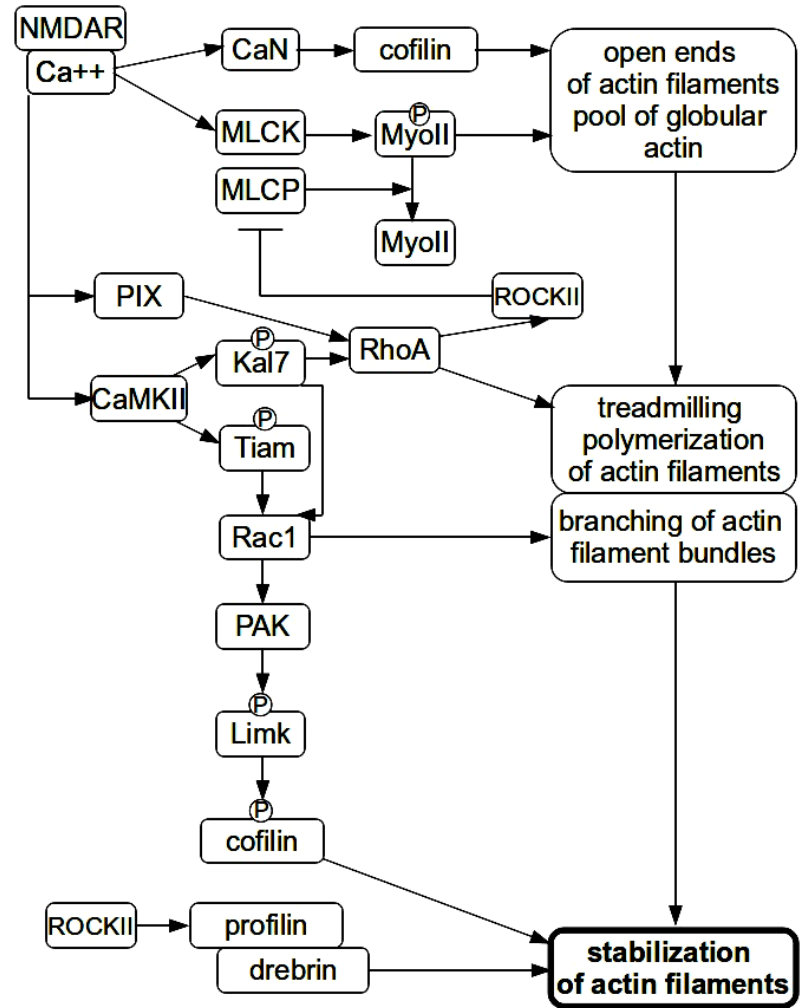

- phosphorylated protein

-I- regulatory interaction, which switch off the reaction.

Figure 2: Schema regulatory interactions, which induced of synaptic activity and provided of dendritic spine cytoarchitecture remodelling. positively affects Racl activation through phosphorylation of GEF Tiaml $1_{\text {Racl }}$ [25], and negatively through activation of SynGap ${ }_{\text {Ras }}$ [26]. The small GTPase Rac1 binds to p21 protein (Cdc42/Rac)-activated kinase1 (PAK), which in turn is recruited to the PSD through the formation of a complex with Shank scaffold protein and GEF PIX $\mathrm{Racl}_{\mathrm{R} /}$ ${ }_{\mathrm{Cd} c 42}$. Thus, PSD scaffold proteins are the original sites for the assembly of functional microdomains, providing a point of physical interaction between effectors, acceptors, and their activators and inhibitors [27].

PPI1 network of dendritic spines reflects the molecular systems, which provides an increase of efficacy of synaptic transmission after an intense and short-term release of neurotransmitter. Such pattern of glutamate receptors activation leads to a transition of spine at the level, corresponding to more efficient synaptic transmission and causes increase of the spine volume. Temporal blocks can be select to describe the behavior of spine molecular system during the transition at the new level. During basal glutamate receptors activation the electrical signal propagates from the spine to the soma within milliseconds. After synaptic potentiation paradigm, in the range of 30 to $60 \mathrm{~s}$, the NMDARmediated $\mathrm{Ca}^{2+}$ input triggers the lateral relocation of AMPAR1/2 from extra synaptic to synaptic zone $[5,6,28]$. Immobilization of AMPAR1/2 in PSD significantly increases the amplitude of synaptic currents [2931]. NMDAR-mediated $\mathrm{Ca}^{2+}$ input is limited by the spine. Available $\mathrm{Ca}^{2+}$ decays with a time constant of about $0.1 \mathrm{~s}$ [32]. $\mathrm{Ca}^{2+}$-dependent activation of CaMKII is also limited by the spine, and decays with a time constant of about $10 \mathrm{~s}$ (self-inhibition). CaMKII integrates the NMDAR-mediated $\mathrm{Ca}^{2+}$ signals [33]. Activation of Rho GTPases switches the short-term signaling of CaMKII for the long-term synapse-specific signaling required for the structural plasticity of spines associated with remodeling of the actin cytoskeleton [34].

The spatiotemporal dynamics of Rho GTPases differ. Activation of RhoA and Cdc42 reaches a maximum in about $30 \mathrm{~s}$ and then activity is reduced and stably maintained for more than $30 \mathrm{~min}$ [35]. Activation of Cdc42 is limited by the activated spine. The high spatial gradient of Cdc42 between the stimulated spine and dendrite is maintained for more than $30 \mathrm{~min}, \mathrm{Cdc} 42$ must be continuously activated in spines during plasticity, and is inactivated immediately after exiting from the spine [34]. Ras activation increases to a maximum within $1 \mathrm{~min}$ after stimulation and returns to a baseline within 15 min [34]. Active RhoA and Ras require about $1 \mathrm{~s}$ to relocate from the spine to the dendrite. Active Rases are propagated about $10 \mu \mathrm{m}$ by diffusion along of the parent dendrite and invade about 10 to 20 neighboring spines [35], active RhoAs are propagated about $5 \mu \mathrm{m}$ [34]. A propagation mechanism of Rho GTPases along the parent dendrite might underlie the association of signals coming out of the neural network to the neighboring spines.

Cascade NMDAR-CaMKII-Cdc42-Pak is specific for spine signal transduction; it cover the time from $1 \mathrm{~s}$ to $30 \mathrm{~min}$. Minor proteins of the actin cytoskeleton are targets of RhoA-ROCK and Rac1/Cdc42PAK. Key proteins involved in spine cytoskeletal reorganization are presented in PPI1 network of dendritic spines. $\mathrm{Ca}^{2+} / \mathrm{calmodulin}$ complexes are key regulators of Ca-sensitive kinases and phosphatases [36]. In the first 2 min after potentiation, activation of the calcineurincofilin, RhoA-ROCK-myosin II cascades leads to the breakdown of existing F-actin, appearance of the open ends of F-actin, and a pool of G-actin, which is necessary for the nucleation of new filaments, their branching, and elongation. Activation cascades of Rac1/Cdc42PAK controls nucleation and branching of the actin network through activation of cortactin-Arp2/3-WAVE/N-WASP functional protein 
complex. Activation of the cascades is also important for stabilizing the new actin network that lasts up to $10 \mathrm{~min}$. Cascades Rac1/ Cdc42-PAK, RhoA-ROCK through the activation of LIM-kinase and phosphorylation of cofilin stabilize new bundles and individual filaments of actin [37], PAK inactivates MLC-kinase, which reduces the activity of myosin II and F-actin tension [38].

PPI1 network of dendritic spines and the description of the processes that are initiated by activation of glutamate receptor reflect the ability of nanosized compartments of the neuron to self-development (the transition to a new level of efficient synaptic transmission) using the own resources. However, for maintaining the new state of spine resources from other compartments of the neuron are needed.

The synthesis of new proteins, occurring in the soma of the neuron, including AMPA receptors [39] is necessary to maintain long-term LTP. LTP in hippocampus is maintained for a long time, but not longer than 30-60 minutes if the synthesis of proteins is blockaded. To maintain the LTP in this initial period a post-translational modification of proteins and the formation of protein-lipid vesicles in the vacuolar system $[40,41]$ are required to a greater extent than protein synthesis. Maintaining the new level of transfer is accompanied by replacement of AMPAR1/2 on AMPAR2/3 subtype [42]. The molecular network (PPI2), mediating these processes is available at: http://wwwmgs. bionet.nsc.ru/mgs/gnw/genenet/viewer/AMPA.html.

The basal stocks of AMPARs $2 / 3$ are in the vacuolar system, where they are included in the vesicles and are delivered to the spines. The movement of proteins between compartments of vacuolar system (endoplasmic reticulum, Golgi apparatus, trans-Golgi network, endosomes) and their delivery to the spine are mediated by small transport vesicles, branching off from a donor compartment and then fuse with an acceptor compartment [43]. The accuracy of vesicle assembly is controlled by its coating proteins. The initiation of the vesicle coating formation and also its branching off from a donor compartment is regulated by small GTPas protein family - ARF (ADPribosylation factor). The transport vesicles with AMPA receptors are delivered to the dendrite where they can be incorporated in the plasmatic membrane of spine dendrite or in the dendritic depot of receptors. Experimental data suggest that the violation of vesicle assemblage does not prevent a transition of spines to new level of the synaptic transmission efficiency but leads to a significant reduce of the synaptic transmission efficiency in the first 20 min after potentiating [44].

\section{Conclusion}

Simulation of complex systems is necessary, because multicomponent molecular system of nerve cell cannot be perceived as a whole without it. We integrated the proteins of pyramidal neurons of the CA1 hippocampal region of the early phase of LTP into a conceptual model. PPI1, PPI2 networks represent a basic set of the molecules and its functional interactions, required for translation of electrochemical signals into the following processes: inclusion of a neuron in the brain networks, recognition of synaptic activity patterns, converting the activation of glutamate receptors in change of the synaptic transmission efficiency/volume of the postsynaptic sides of synapses. Dendritic spines are a dynamic structure. GeneNet computer system can reflect the organization of the protein-protein interaction networks in one of the possible states. Reconstructed networks focus mostly on static interactions, mapping the set of potential interactions among proteins of neuronal compartment. Some interactions are transient and may occur only under specific conditions. In this article temporal dynamics of the process are brought in the form of a script. Perhaps PPI1, PPI2 networks can become fragments (subsystems) of complete electronic circuits of certain type neurons.

\section{References}

1. Lin L, Shen S, Jiang P, Sato S, Davidson BL, et al. (2010) Evolution of alternative splicing in primate brain transcriptomes. Hum Mol Genet 19: 2958-2973.

2. Penn AC, Balik A, Wozny C, Cais O, Greger IH (2012) Activity-mediated AMPA receptor remodeling, driven by alternative splicing in the ligand-binding domain. Neuron 76: 503-510.

3. Barbosa-Morais NL, Irimia M, Pan Q, Xiong HY, Gueroussov S, et al. (2012) The evolutionary landscape of alternative splicing in vertebrate species. Science 338: 1587-1593.

4. Kandel ER (2001) The molecular biology of memory storage: a dialogue between genes and synapses. Science 294: 1030-1038.

5. Heine M, Groc L, Frischknecht R, Béïque JC, Lounis B, et al. (2008) Surface mobility of postsynaptic AMPARs tunes synaptic transmission. Science 320 : 201-205.

6. Makino H, Malinow R (2009) AMPA receptor incorporation into synapses during LTP: the role of lateral movement and exocytosis. Neuron 64: 381-390.

7. Sheng M, Kim MJ (2002) Postsynaptic signaling and plasticity mechanisms Science 298: 776-780

8. Bourne JN, Harris KM (2008) Balancing structure and function at hippocampa dendritic spines. Annu Rev Neurosci 31: 47-67.

9. Martin SJ, Grimwood PD, Morris RG (2000) Synaptic plasticity and memory: an evaluation of the hypothesis. Annu Rev Neurosci 23: 649-711.

10. Pastalkova E, Serrano P, Pinkhasova D, Wallace E, Fenton AA, et al. (2006) Storage of spatial information by the maintenance mechanism of LTP. Science 313: 1141-1144

11. Pocklington AJ, Cumiskey M, Armstrong JD, Grant SG (2006) The proteomes of neurotransmitter receptor complexes form modular networks with distributed functionality underlying plasticity and behaviour. Mol Syst Biol 2: 2006.

12. Kolpakov FA, Ananko EA (1999) Interactive data input into the GeneNet database. Bioinformatics 15: 713-714.

13. Ananko EA, Podkolodny NL, Stepanenko IL, Podkolodnaya OA, Rasskazov DA, et al. (2005) GeneNet in 2005. Nucleic Acids Res 33: D425-D427.

14. Ananko EA, Podkolodny NL, Stepanenko IL, Ignatieva EV, Podkolodnaya OA et al. (2002) GeneNet: a database on structure and functional organisation of gene networks. Nucleic Acids Res 30: 398-401.

15. Stepanenko I, Kolchanov N (2003) Apoptosis gene network: description in the GeneNet and TRRD databases. Ann N Y Acad Sci 1010: 16-18.

16. Valtschanoff JG, Weinberg RJ (2001) Laminar organization of the NMDA receptor complex within the postsynaptic density. J Neurosci 21: 1211-1217.

17. Chen X, Winters C, Azzam R, Li X, Galbraith JA, et al. (2008) Organization of the core structure of the postsynaptic density. Proc Natl Acad Sci U S A 105 : 4453-4458.

18. Shi S, Hayashi Y, Esteban JA, Malinow R (2001) Subunit-specific rules governing AMPA receptor trafficking to synapses in hippocampal pyramidal neurons. Cell 105: 331-343.

19. Schiller J, Schiller Y, Clapham DE (1998) NMDA receptors amplify calcium influx into dendritic spines during associative pre- and postsynaptic activation. Nat Neurosci 1: 114-118.

20. Tomita S, Stein V, Stocker TJ, Nicoll RA, Bredt DS (2005) Bidirectional synaptic plasticity regulated by phosphorylation of stargazin-like TARPs. Neuron 45 269-277.

21. Choi J, Ko J, Park E, Lee JR, Yoon J, et al. (2002) Phosphorylation of stargazin by protein kinase $A$ regulates its interaction with PSD-95. J Biol Chem 277 12359-12363.

22. lida J, Ishizaki H, Okamoto-Tanaka M, Kawata A, Sumita K, et al. (2007) Synaptic scaffolding molecule alpha is a scaffold to mediate N-methyl-Daspartate receptor-dependent RhoA activation in dendrites. Mol Cell Biol 27 4388-4405. 
Citation: Proskura AL, Ratushnyak AS, Zapara TA (2014) The Protein-Protein Interaction Networks of Dendritic Spines in the Early Phase of LongTerm Potentiation. J Comput Sci Syst Biol 7: 040-044. doi:10.4172/jcsb.1000136

23. Schubert $V$, Da Silva JS, Dotti CG (2006) Localized recruitment and activation of RhoA underlies dendritic spine morphology in a glutamate receptor-dependent manner. J Cell Biol 172: 453-467.

24. Tejada-Simon MV, Villasana LE, Serrano F, Klann E (2006) NMDA receptor activation induces translocation and activation of Rac in mouse hippocampal area CA1. Biochem Biophys Res Commun 343: 504-512.

25. Fleming IN, Elliott CM, Buchanan FG, Downes CP, Exton JH (1999) $\mathrm{Ca}^{2+}$ calmodulin-dependent protein kinase II regulates Tiam1 by reversible protein phosphorylation. J Biol Chem 274: 12753-12758.

26. Carlisle HJ, Manzerra P, Marcora E, Kennedy MB (2008) SynGAP regulates steady-state and activity-dependent phosphorylation of cofilin. J Neurosci 28 : 13673-13683.

27. Park E, Na M, Choi J, Kim S, Lee JR, et al. (2003) The Shank family of postsynaptic density proteins interacts with and promotes synaptic accumulation of the beta PIX guanine nucleotide exchange factor for Rac1 and Cdc42. J Biol Chem 278: 19220-19229.

28. Petrini EM, Lu J, Cognet L, Lounis B, Ehlers MD, et al. (2009) Endocytic trafficking and recycling maintain a pool of mobile surface AMPA receptors required for synaptic potentiation. Neuron 63: 92-105.

29. Petersen CC, Malenka RC, Nicoll RA, Hopfield JJ (1998) All-or-none potentiation at CA3-CA1 synapses. Proc Natl Acad Sci U S A 95: 4732-4737.

30. O'Connor DH, Wittenberg GM, Wang SS (2005) Graded bidirectional synaptic plasticity is composed of switch-like unitary events. Proc Natl Acad Sci U S A 102: 9679-9684.

31. Bredt DS, Nicoll RA (2003) AMPA receptor trafficking at excitatory synapses. Neuron 40: 361-379.

32. Sabatini BL, Oertner TG, Svoboda K (2002) The life cycle of $\mathrm{Ca}(2+)$ ions in dendritic spines. Neuron 33: 439-452.

33. Lee SJ, Escobedo-Lozoya Y, Szatmari EM, Yasuda R (2009) Activation of
CaMKII in single dendritic spines during long-term potentiation. Nature 458 299-304.

34. Murakoshi H, Wang H, Yasuda R (2011) Local, persistent activation of Rho GTPases during plasticity of single dendritic spines. Nature 472: 100-104.

35. Harvey CD, Yasuda R, Zhong H, Svoboda K (2008) The spread of Ras activity triggered by activation of a single dendritic spine. Science 321: 136-140.

36. Wayman GA, Lee YS, Tokumitsu H, Silva AJ, Soderling TR (2008) Calmodulinkinases: modulators of neuronal development and plasticity. Neuron 59: 914 931.

37. Szczepanowska J (2009) Involvement of Rac/Cdc42/PAK pathway in cytoskeletal rearrangements. Acta Biochim Pol 56: 225-234.

38. Chew TL, Masaracchia RA, Goeckeler ZM, Wysolmerski RB (1998) Phosphorylation of non-muscle myosin II regulatory light chain by p21-activated kinase (gamma-PAK). J Muscle Res Cell Motil 19: 839-854.

39. Abraham WC, Williams JM (2008) LTP maintenance and its protein synthesisdependence. Neurobiol Learn Mem 89: 260-268.

40. Brown TC, Correia SS, Petrok CN, Esteban JA (2007) Functional compartmentalization of endosomal trafficking for the synaptic delivery of AMPA receptors during long-term potentiation. J Neurosci 27: 13311-13315.

41. Brown TC, Tran IC, Backos DS, Esteban JA (2005) NMDA receptor-dependent activation of the small GTPase Rab5 drives the removal of synaptic AMPA receptors during hippocampal LTD. Neuron 45: 81-94.

42. Newpher TM, Ehlers MD (2008) Glutamate receptor dynamics in dendritic microdomains. Neuron 58: 472-497.

43. Hinners I, Tooze SA (2003) Changing directions: clathrin-mediated transport between the Golgi and endosomes. J Cell Sci 116: 763-771.

44. Malakchin IA, Proskura AL, Zapara TA, Ratushnyak AS (2012) Influence of transport vesicles assembly to preserve the effectiveness of the synaptic transmission. Vestnik NGU (russian) 10: 14-20. 